\title{
Double Charged Surface Layers in Lead Halide
}

\section{Perovskite Crystals}

Smritakshi P. Sarmah ${ }^{1 \dagger}$, Victor M. Burlakov ${ }^{2 \dagger}$, Emre Yengel ${ }^{1 \dagger}$, Banavoth Murali ${ }^{l}$, Erkki Alarousu ${ }^{l}$, Ahmed M El-Zohry ${ }^{1}$, Chen Yang ${ }^{1}$, Mohd S. Alias ${ }^{3}$, Ayan Zhumekenov ${ }^{l}$, Makhsud I. Saidaminov ${ }^{1}$, Namchul Chao, Nimer Wehbe ${ }^{4}$, Somak Mitra ${ }^{5}$, Idris Ajia ${ }^{5}$, Sukumar Dey ${ }^{1}$, Ahmed Mansur $^{1}$, Aram Amassian ${ }^{1}$, Iman S Roqan ${ }^{5}$, Boon S. Ooi ${ }^{3}$, Alain Goriely ${ }^{2}$, Osman M. Bakr ${ }^{1}$, Omar F. Mohammed ${ }^{1}$

${ }^{1}$ King Abdullah University of Science and Technology, KAUST Solar Center, Division of Physical Sciences and Engineering, Thuwal 23955-6900, Kingdom of Saudi Arabia

${ }^{2}$ Mathematical Institute, University of Oxford, Woodstock Road, Oxford OX2 6GG, United Kingdom

${ }^{3}$ King Abdullah University of Science and Technology, Photonics Laboratory, Computer, Electrical and Mathematical Sciences and Engineering Division, Thuwal 23955-6900, Kingdom of Saudi Arabia

${ }^{4}$ King Abdullah University of Science and Technology, Imaging and Characterization Laboratory, Thuwal 23955-6900, Kingdom of Saudi Arabia

${ }^{5}$ King Abdullah University of Science and Technology, Semiconductor and Material Spectroscopy Laboratory, Material Science \& Engineering Division, Thuwal 23955-6900, Kingdom of Saudi Arabia 
KEYWORDS. Nanometer surface layer, Lead halide perovskite crystal, One vs two photonexcitation and Ion migration

ABSTRACT. Understanding defect chemistry, particularly ion migration, and its significant effect on the surface's optical and electronic properties is one of the major challenges impeding the development of hybrid perovskite-based devices. Here, using both experimental and theoretical approaches, we demonstrate that the surface layers of the perovskite crystals may acquire a high concentration of positively charged halide vacancies with the complementary negatively charged halide ions pushed to the surface. This charge separation near to the surface generate an electric field that can induce a shift in the optical band gap of the surface layers to higher energy compared to the bulk counterpart. We found that the charge separation, electric field and the amplitude of shift in the bandgap strongly depend on the halides and organic moieties of perovskites crystals. Our findings reveal the peculiarity of surface effects that is currently limiting the application of perovskite crystals and more importantly explain their origins, thus enabling viable surface passivation strategies to remediate them.

\section{TOC Graphic}

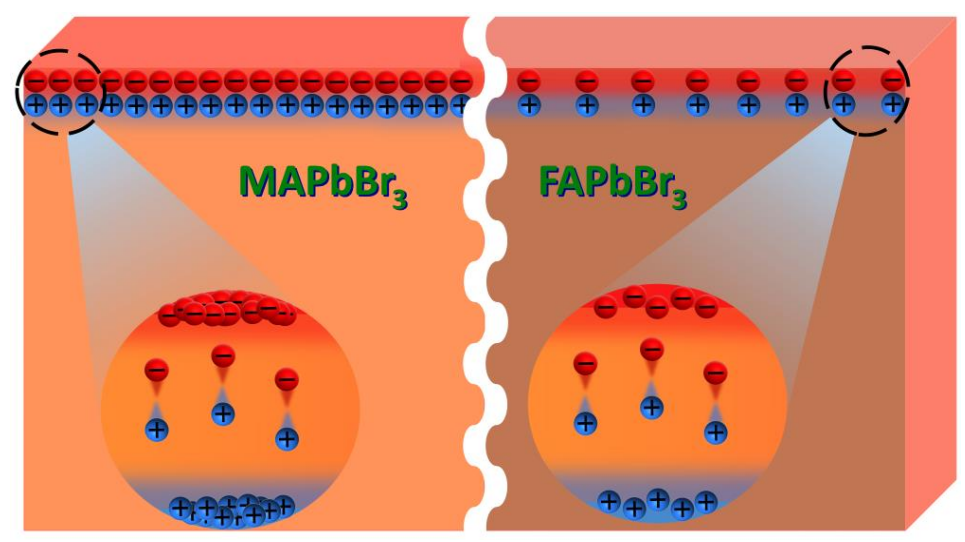


Organic-inorganic hybrid halide perovskites have recently become one of the most important classes of photoactive materials in the field of photovoltaics due to their remarkable optoelectronic properties $^{1-4}$ and cost-effective fabrication processes ${ }^{5,6}$. Within a short period of time, the power conversion efficiency (PCE) of perovskite-based devices has increased from $3.8 \%{ }^{7}$ to $>20 \%{ }^{4,8}$ which represents a significant breakthrough in the development cycle of a photovoltaic technology.

Although there is an outstanding photovoltaic performance of these devices based on the polycrystalline perovskite films, these devices still severely suffer from the undesirable surface traps that significantly affect the device operation, suggesting that there is still a room for some major performance improvements in these working devices. Recently, the discovery of high quality perovskite crystals $^{9-11}$ with much lower defect concentration, higher charge-carrier mobility, longer carrier life time ${ }^{11}$, and longer diffusion lengths ${ }^{1,12}$ has led to better devices ${ }^{13}$ compared to polycrystalline films. ${ }^{14}$ However, it has recently been suggested that the optical and electronic properties of the single-crystal surface are significantly different from those in the bulk $^{15}$ (see Fig.1) due to surface disorder and halide migration, effects that are typical for polycrystalline films. ${ }^{16-21}$ If this is the case, then the single crystal device would suffer from resistive losses and high leakage current in solar cells. ${ }^{22}$ Therefore, understanding the surface layer properties of perovskite crystals is important for finding suitable surface passivation ${ }^{23}$ to further improve device performance.

Here, we reveal through experimental and theoretical investigations the origin of the main differences in the behavior of single crystal surface compared to its bulk. We show that these differences are due to the electric field generated near the surface of the crystals via spontaneous 
separation of negatively charged halides ions and positively charged vacancies. Such charge separation results from the formation of Frenkel defects ${ }^{24}$ near the surface with halides ions pushed to the single crystal surfaces due to high elastic strain and with the remaining vacancies kept in the proximity to these surfaces by Coulomb interactions with the ions. We combine experimental studies of Secondary ion mass spectrometry (SIMS),Scanning electron microscopy (SEM) and the photoluminescence (PL) after one photon (surface layer PL) and with two-photon (bulk PL) with theoretical arguments to explain the undesirable behavior of the single crystal surfaces. In addition, our work not only shows that ionic defects can strongly influence the nature of the interfaces and surfaces of perovskites, but also suggests possible ways of improving the material properties by surfaces passivation.

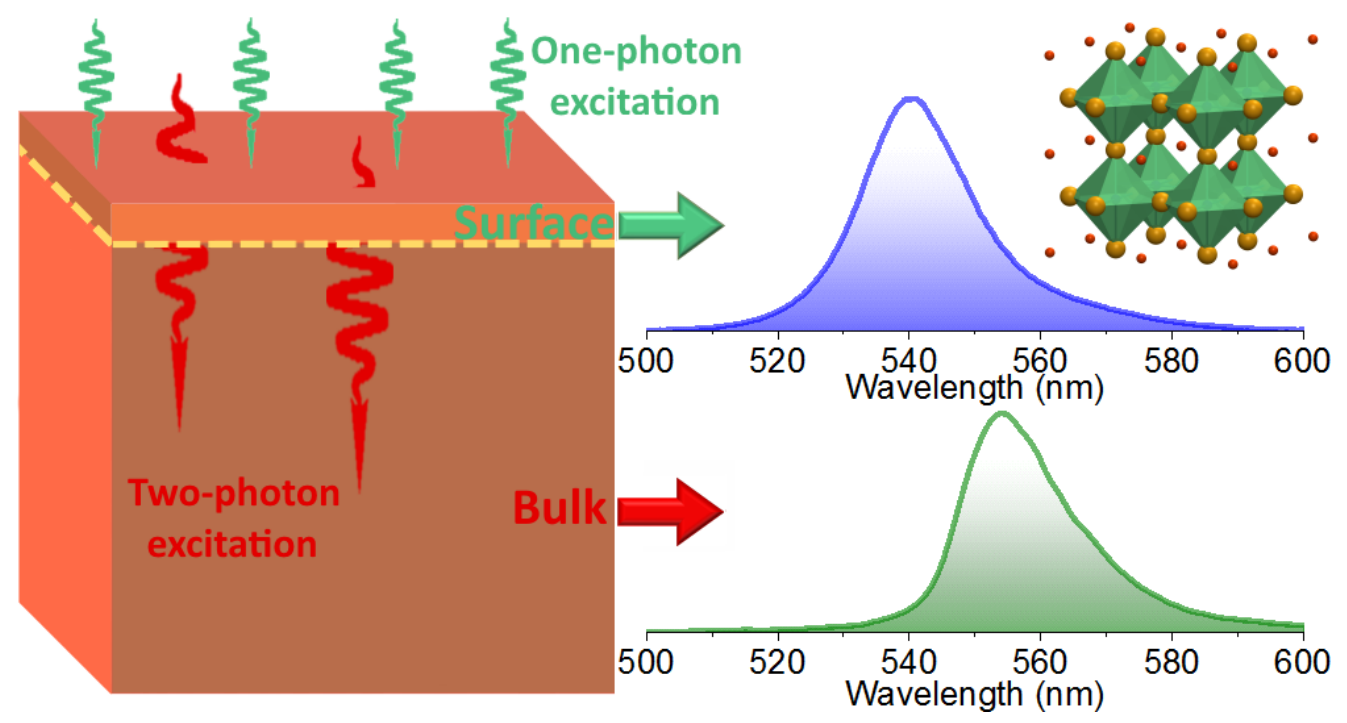

Figure 1. Schematic diagram of difference in the of photoluminescence behaviour of surface and bulk in $\mathrm{MAPbBr}_{3}$ single crystal obtained under one-photon (1p) and two-photon (2p) excitations. 
The single crystals used in the current study were synthesised by using inverse temperature technique (see supporting information). The photoluminescence (PL) in organo-lead halide perovskite single crystals obtained by either one photon (1p) excitation above the band gap, or by two-photon (2p) excitation below the band gap exhibit striking difference in the positions of the PL line (see Fig.2 (a)). After the correction for the light reabsorption (see the detail below and the supporting information as well), the $1 \mathrm{p}$ and $2 \mathrm{p}$ experiments result in two different peaks for $\mathrm{MAPbBr}_{3}$ positioned at $540 \mathrm{~nm}(2.3 \mathrm{eV})$ and $555 \mathrm{~nm}(2.24 \mathrm{eV})$, respectively. Interestingly, corresponding PL spectra for $\mathrm{FAPbBr}_{3}$ single crystal are centred at $560 \mathrm{~nm}(2.2 \mathrm{eV})$ for both $1 \mathrm{p}$ and 2p. In contrast, due to the low activation energy of $\mathrm{I}^{-}$in both $\mathrm{MAPbI}_{3}$ and $\mathrm{FAPbI}_{3}$ crystals exhibit striking difference in the positions and the shape of the PL line after $1 p$ and $2 p$ excitations. For instance, for $\mathrm{MAPbI}_{3}$ and $\mathrm{FAPbI}_{3}$ the former shows the PL spectrum centred at $774 \mathrm{~nm}(1.61 \mathrm{eV})$ for $1 \mathrm{p}$ and $793 \mathrm{~nm}(1.56 \mathrm{eV})$ for $2 \mathrm{p}$, while the PL spectra for $\mathrm{FAPbI}_{3}$ are centred at $815 \mathrm{~nm}(1.50 \mathrm{eV})$ and $843 \mathrm{~nm}(1.47 \mathrm{eV})$ for the $1 \mathrm{p}$ and $2 \mathrm{p}$, (Fig.S1) respectively. Notably, these aforementioned values of the PL lines are after the correction for re-absorption of light inside the single crystals (see below).

It would be reasonable to assume that the difference in the PL spectra for each type of excitation (1p or $2 p)$ is due to the difference in the bulk and surface layer properties. Indeed, the PL obtained with excitation above the band gap characterises mainly the surface layer with thickness determined by the light penetration depth and it is normally few hundreds of nanometres ${ }^{25}$. In contrast, the PL spectra collected after $2 p$ excitation is generated throughout the entire medium due to the long light penetration depth and it mainly reflects the bulk properties ${ }^{26}$. It should be noted here that, if the thickness of the crystal is larger than the carrier diffusion length ${ }^{11}$; the high-energy photons cannot escape from the interior bulk due to the large absorption 
coefficient, but they are likely re-absorbed and re-emitted as lower-energy photons ${ }^{27}$. The reemitted lower energy photon are observed at $2.1 \mathrm{eV}(580 \mathrm{~nm})$ and $2.13 \mathrm{eV}(590 \mathrm{~nm})$ for $\mathrm{MAPbBr}_{3}$ and $\mathrm{FAPbBr}_{3}$ single crystal, respectively. Therefore, by considering the correction factor (see supporting information for details), using the absorption coefficient ${ }^{28}$ and diffusion length $^{9-11}$ the emission peak at $580 \mathrm{~nm}$ for $\mathrm{MAPbBr}_{3}$ significantly reduced, and new PL position for $2 p$-excitation was observed at $555 \mathrm{~nm}$ (Fig. 2 (a)). This observation has been confirmed by measuring the thin $\mathrm{MAPbBr}_{3}$ single crystals, close to the diffusion length of the charge carrier (i.e there is no possibility of reabsorption) which gives directly PL spectrum at $555 \mathrm{~nm}$ upon 2pexcitation (Fig. S2 and its corresponding SEM images in Fig. S3). It should be noted the PL spectrum for the thick and thin $\mathrm{MAPbBr}_{3}$ is still located at $540 \mathrm{~nm}$ upon $1 \mathrm{p}$-excitation .So there is almost $15 \mathrm{~nm}$ spectral shift when we go from $1 \mathrm{p}$ to $2 \mathrm{p}$ excitation in case of $\mathrm{MAPbBr}_{3}$. On the other hand there is almost no spectral shift in case of $\mathrm{FAPbBr}_{3}$ between $1 \mathrm{p}$ and $2 \mathrm{p}$ excitation after correction for the re-absorption process, indicating that the ion migration and ion redistribution is very small if any at room temperature. This important observation has been supported by several experiments (see later sections)

The main problem we address here is about the possible mechanism responsible for the shift of the PL line in the surface layer relative to the bulk in $\mathrm{MAPbBr}_{3}$ compared to $\mathrm{FAPbBr}_{3}$. This effect can be due to electrostatic interactions, e.g. electric field causing the shift of the band gap either directly ${ }^{29-32}$ or via electrostriction effect ${ }^{33,34}$.The origin of an electric field in the surface layer can be understood as follows.

At any finite temperature crystal lattices, especially in ionic crystals, generate point defects (Frenkel pairs) to increase the entropy at the expense of some increase in the internal energy ${ }^{35}$. At thermodynamic equilibrium these two contributions to free energy balance each other 
resulting in a finite concentration of point defects in the crystal. In the case of the organo-lead halide perovskite the main point implies that the halide ions tend to go to the crystal grain surfaces $^{36}$ to reduce the free energy. In particular the spontaneous formation of defects near the surface leads to halide ions migrating to the surface while halide vacancies penetrate the structure away from the surface defects are halide ions and vacancies ${ }^{19,37-39}$. Iodine vacancy is the dominant diffusing defect due to its low formation energy and the low diffusion barrier ${ }^{19,20,}$ 24, 40. According to DFT studies the presence of halide ions in the perovskite structure is energetically costly. This
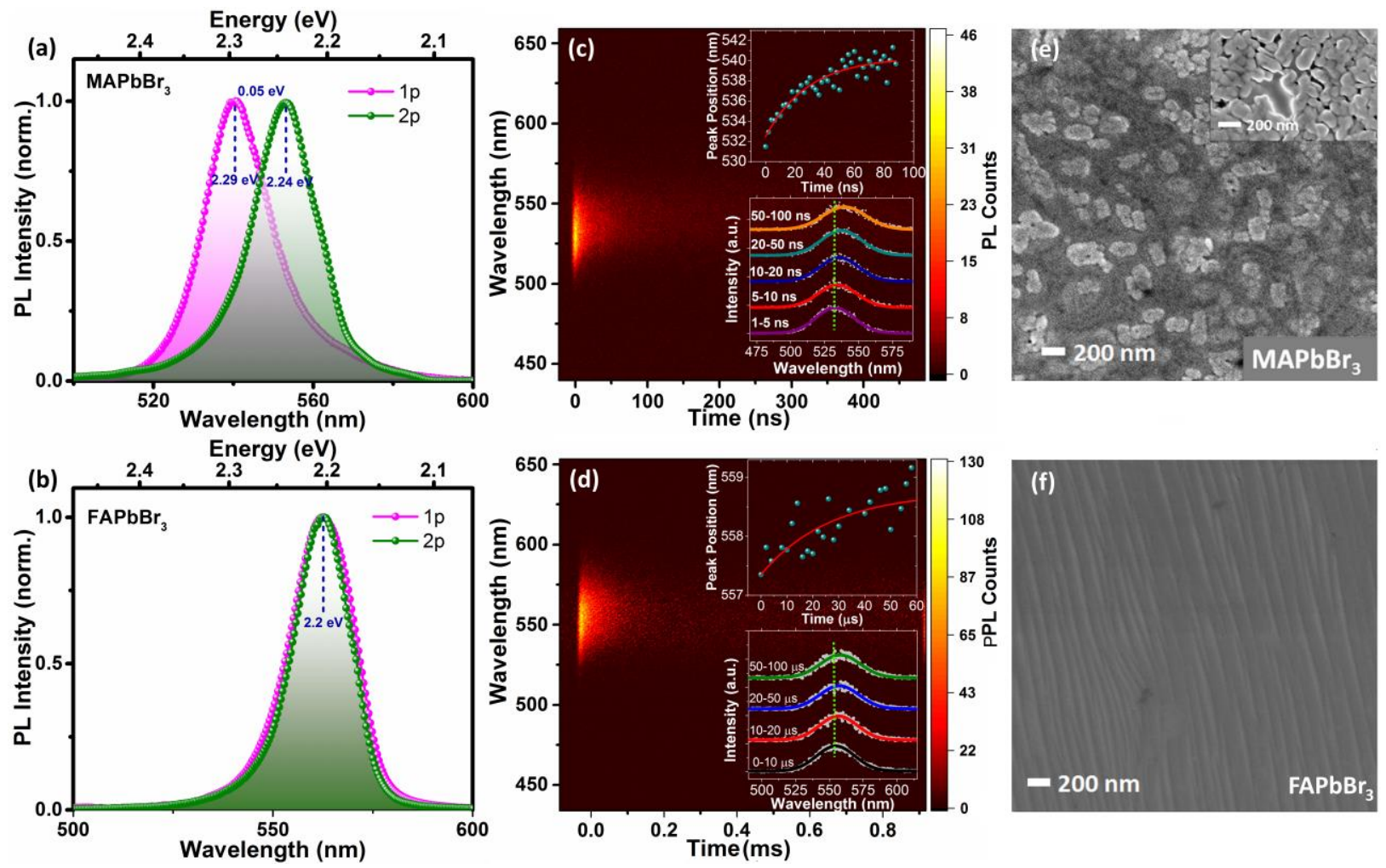

Figure 2. PL spectra of single crystals $\mathrm{MAPbBr}_{3}$ (a) and $\mathrm{FAPbBr}_{3}(\mathrm{~b})$ obtained after $1 \mathrm{p}$ and $2 \mathrm{p}$ excitation as indicated in the figure; Time-resolved 1p PL spectra obtained at different time delay as indicated on each panel for $\mathrm{MAPbBr}_{3}$ (c) and $\mathrm{FAPbBr}_{3}$ (d) (The PL positons were corrected with respect 
to the steady state PL position).; and SEM micrographs showing the single crystal surfaces of $\mathrm{MAPbBr}_{3}$ (e) and $\mathrm{FAPbBr}_{3}(\mathrm{f})$ respectively.

This defect formation results in macroscopic charge separation, i.e. it generates a macroscopic electric field $E(x)$ perpendicular to the surface that can attract the vacancies to the crystal surface, and can screen this field thus confining it to the surface layer. Interestingly, in case of $\mathrm{FAPbBr}_{3}$ such ion migration is very minimal, therefore, there is no shift in PL between $1 p$ and $2 p$ excitation. This can be attributed to the larger size of the FA and its stronger hydrogen bond with $\mathrm{Br}^{-}$, which can minimize the motion of the cation unit and subsequently suppress or hinder the ion migration to the surface ${ }^{41}$.

The picosecond (ps) time-resolved 1p PL spectra was recorded using Streak Camera with ps temporal resolution and broadband capability, which clearly demonstrate that the PL lifetime of the surface of $\mathrm{MAPbBr}_{3}$ single crystal is very different compared to $\mathrm{FAPbBr}_{3}$ counterparts. The PL spectra shift at early time (0-100 ns scale) is about $8 \mathrm{~nm}$ and $3 \mathrm{~nm}$ for $\mathrm{MAPbBr}_{3}$ and $\mathrm{FAPbBr}_{3}$ single crystals, respectively, indicating the photo induced changes including ion redistribution or/and migration is much larger in the case of $\mathrm{MAPbBr}_{3}$ single crystal (see Fig. 2 (c) and 2 (d)). It is worth pointing out that the PL spectral shift at early time is recently attributed to the carrier diffusion from the surface to the bulk ${ }^{42}$. However, if this is the only origin for such spectral shift, we should observe similar shift (if not more) in the case of $\mathrm{FAPbBr}_{3}$ single crystals ${ }^{11}$ but this is not the case. This would mean that we do have more ion migration or redistribution in the case of $\mathrm{MAPbBr}_{3}$ single crystal compared to $\mathrm{FAPbBr}_{3}$. More specifically, it was found that under the same experimental conductions, the lifetime is almost one order of magnitude shorter in the case of $\mathrm{MAPbBr}_{3}$ single crystal as compared to $\mathrm{FAPbBr}_{3}$ (see Fig.S4), providing another piece of 
evidence that that the number of trap states/vacancies due to ion migration and re-distribution are much lesser in the case of $\mathrm{FAPbBr}_{3}$ than that for $\mathrm{MAPbBr}_{3}$.

Recent reports have clearly indicated the passage of ions to the surface through the grain boundaries ${ }^{43,44}$ So the grain boundaries plays a critical role in ion migration. To further prove the ion migration in $\mathrm{MAPbBr}_{3}$, we conducted the surface-morphology mapping using highresolution scanning electron microscopy; and it has been found that of the surface of $\mathrm{MAPbBr}_{3}$ single crystals show more nano and micro grain boundaries ${ }^{45}$, which is very possibly due to the surface hydration and disorder. A detailed investigation using the state of art atomic scale resolution scanning tunneling microscope (STM), on the restructuring of single crystals are described elsewhere ${ }^{45}$. Hence, the greater part of grains on the $\mathrm{MAPbBr}_{3}$ can act as rattling centers to drive the ions towards the surface. On the contrary, $\mathrm{FAPbBr}_{3}$ single crystal surface showed characteristic layered crystal surface with no grain boundary formations, suggesting negligible ion migration (see Fig. 2(e) and (f)).

To further substantiate the ion migration through the bulk, the ps time-resolved $2 p$ PL was recorded using Streak Camera in $\mathrm{MAPbBr}_{3}$ single crystal. We found that there is no PL spectra shift at early time to us scale in $\mathrm{MAPbBr}_{3}$ single crystals which confirms that the bulk of the single crystal is intake with the bromide ion and there is no ion migration or redistribution inside.
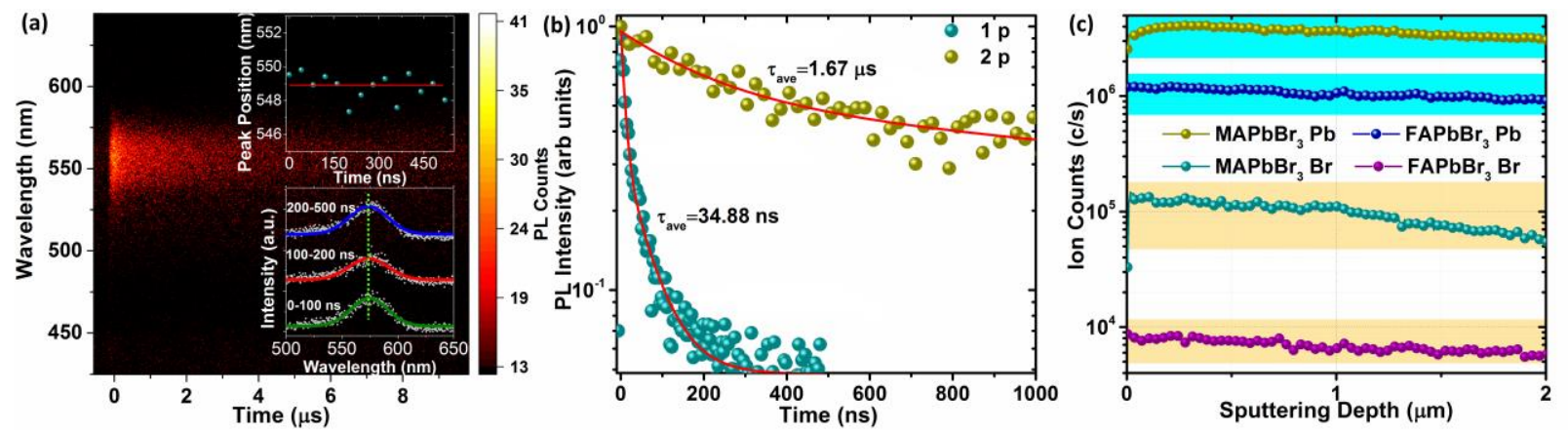
Figure 3. Time-resolved PL spectra obtained at different time scale for $\mathrm{MAPbBr}_{3}$ upon $2 \mathrm{p}$ excitation (a); PL decay of $1 \mathrm{p}$ and $2 \mathrm{p}$ excitation of $\mathrm{MAPbBr}_{3}$ (b) SIMS depth profiling performed on both $\mathrm{MAPbBr}_{3}$ and $\mathrm{FAPbBr}_{3}$ single crystals. Only $\mathrm{Br}$ and $\mathrm{Pb}$ traces are shown for better comparison (c).

To put further evidences of ion migration from the surface towards the bulk we have also performed the Secondary ion mass spectrometry (SIMS). In this experiment, the depth profiling $(0 \mathrm{~nm}-2 \mu \mathrm{m})$ of ion concentrations especially the $\mathrm{Br}^{-}$and $\mathrm{Pb}^{+2}$ from the top surface to the bulk is quantified. More traces of halide ions were detected on surface of $\mathrm{MAPbBr}_{3}$ single crystal further fortifying the pronounced ion migration (See Fig. 3(c)). However, on the other hand, $\mathrm{FAPbBr}_{3}$, the ions distribution as we go from the surface to the bulk is almost uniform, indicating minimal or negligible ion migration. This can be attributed to the larger size of the FA and the stronger hydrogen bond between FA and Br-, which can hinders the motion of the cation unit and subsequently suppress the ion migration to the surface ${ }^{41}$.

To correlate the ion migration and the PL blue shift between the $1 \mathrm{p}$ and $2 \mathrm{p}$, in $\mathrm{MAPbBr}_{3}$ theoretical calculations were performed and compared with the experimental results. We first analyze the spatial variation of the electric field and concentration of vacancies $n_{v}(x)$. In thermal equilibrium, the distribution of vacancies is controlled by the balance between the diffusion and drift currents of vacancies in the electric field $E(x)$ produced by halide ions on the surface (see Eq. (S1) in the Supporting Information).

This balance equation can be solved taking into account charge conservation together with the Einstein relation between the diffusion coefficient and mobility to obtain

$$
n_{V}(x)=\frac{2 \varepsilon_{M} k T \cdot n_{S}^{2} \cdot e^{2}}{\left(e^{2} \cdot n_{S} \cdot x+2 \varepsilon_{M} k T\right)^{2}}
$$


where ${ }^{n_{s}}$ is the surface concentration of halide ions, coefficient is the vacancy charge, $k$ is Boltzman's constant, $T$ is temperature, and $\varepsilon_{M}$ is the static dielectric constant of the material. Assuming that the band gap of the material in the surface layer (hence the PL frequency shift $\Delta$ ) is proportional to the squared-average electric field, we have

$$
\Delta \propto\left\langle E^{2}\right\rangle=\frac{2 e^{2} k T\left(n_{S}\right)^{2}}{\varepsilon_{M}\left(e^{2} n_{S} x_{0}+2 \varepsilon_{M} k T\right)}
$$

where $x_{0}$ is the characteristic penetration depth of light. This expression indicates that the blue shift of the PL line can be relatively high for $1 \mathrm{p}$ excitation, which is characterized by a small light penetration depth $x_{0}$, and much lower for the $2 \mathrm{p}$ excited PL due to much higher $x_{0}$ values.
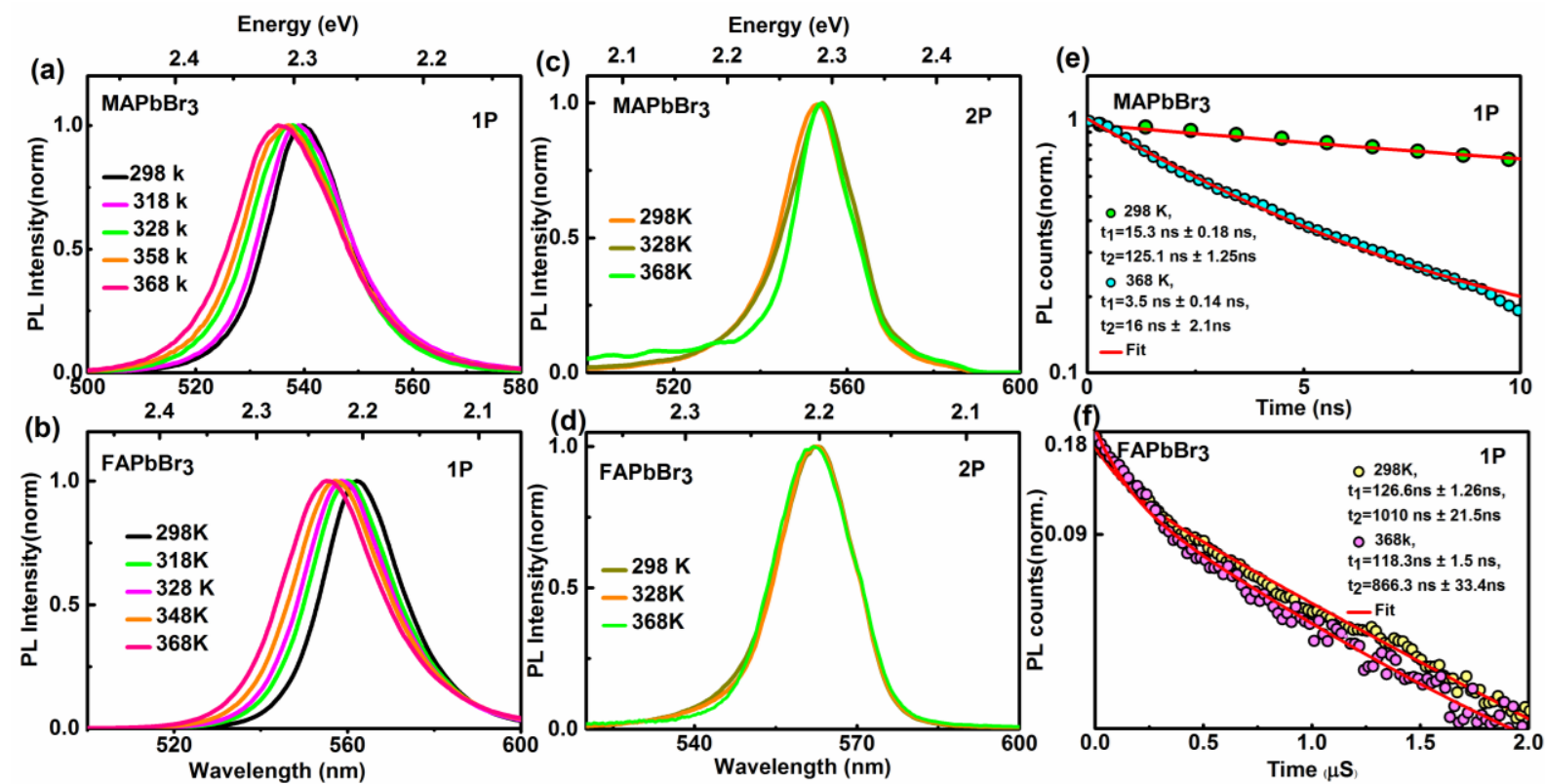

Figure 4. PL spectra for different temperatures of single crystal of $\mathrm{MAPbBr}_{3}$ and $\mathrm{FAPbBr}_{3}$ obtained under $1 \mathrm{p}$ excitation (shown in corresponding panels); $2 \mathrm{p}$ excitation (c) and (d) and temperature dependent $\mathrm{PL}$ decay of $\mathrm{MAPbBr}_{3}(\mathrm{e})$ and $\mathrm{FAPbBr}_{3}(\mathrm{f})$. 
We have also done the temperature dependent PL measurement and noticed that the increase in temperature causes a clear increase in the blue shift for all single crystals with 1p excitation (see Fig. 4(a) and (b) and S5). At higher temperature upon 1p-excitation, a clear PL spectral shift of $\sim 5 \mathrm{~nm}$ and $7 \mathrm{~nm}$ is observed in $\mathrm{MAPbBr}_{3}$ and $\mathrm{FAPbBr}_{3}$, respectively (See Fig. 4(a) and 4(b)). Therefore it is now fairly established that temperature facilities the ion migration in both the MA and FA crystal. Interestingly the blue shift with temperature when compared with respect to bulk PL (2p) for $\mathrm{MAPbBr}_{3}(555 \mathrm{~nm})$ and $\mathrm{FAPbBr}_{3}(560 \mathrm{~nm})$, we found that the shift is $20 \mathrm{~nm}$ for $\mathrm{MAPbBr}_{3}$ and $8 \mathrm{~nm}$ for $\mathrm{FAPbBr}_{3}$. Hence it is also clear that temperature can induce the ion migration in FA however, when compared to MA, it is minimal. In sharp contrast, almost no shift is observed for $2 \mathrm{p}$-excitation, providing another strong piece of evidence that the ions are more concentrated on the surface of the single crystal compared to the bulk.

The ionic conductivity extracted using the complex impedance Nyquist plots, has clearly demonstrated the increase of ionic conductivity with temperature ${ }^{46}$. The migration of ions can cause the charge accumulated at the specific regions or near the interface of the device. The $\mathrm{MAPbBr}_{3}$ single crystal device showed a dependence of ionic conductivity (see Fig. S7) upon temperature, suggesting the temperature induced ion migration due to the low formation energies of interstitial halide ions and/or halide vacancies.

To correlate the temperature induced ion migration/redistribution theoretical calculations were performed and compared with the experimental results. According to Eq. (3), the blue shift of the PL line in the surface layer relative to the bulk depends on temperature, which offers an independent way to validate our model. As can be seen in Fig. 4(a), the increase in temperature causes a clear increase in the blue shift for all singe crystals. This effect can be compared to our theoretical predictions of the blue shift value assuming that the proportionality coefficient 
between the PL shift and the squared-average electric field in Eq. (3) remains constant in the relevant temperature range.

To describe the temperature dependence of the blue shift $\Delta$, we have to find equilibrium values of $n_{s}$ as a function of temperature. This value is obtained by analysing the free energy of the system that obtained in the mean-field approximation by neglecting small repulsive interactions between individual ions on the surface and between individual vacancies in the bulk (see Eq. (S7) in the Supporting Information). The minimization of the free energy with respect to $n_{S}$ gives an equation, which can be solved for $n_{s}$ in the limit $n_{s} \ll N_{s 0}$ (see Section 2 in Supporting Information) to give

$$
n_{s} \approx\left(\frac{2 \varepsilon_{M} k T \cdot N_{v 0} N_{s 0}}{e^{2}}\right)^{1 / 3} \exp \left[-\frac{\varepsilon_{v 0}+2 k T}{3 k T}\right]
$$



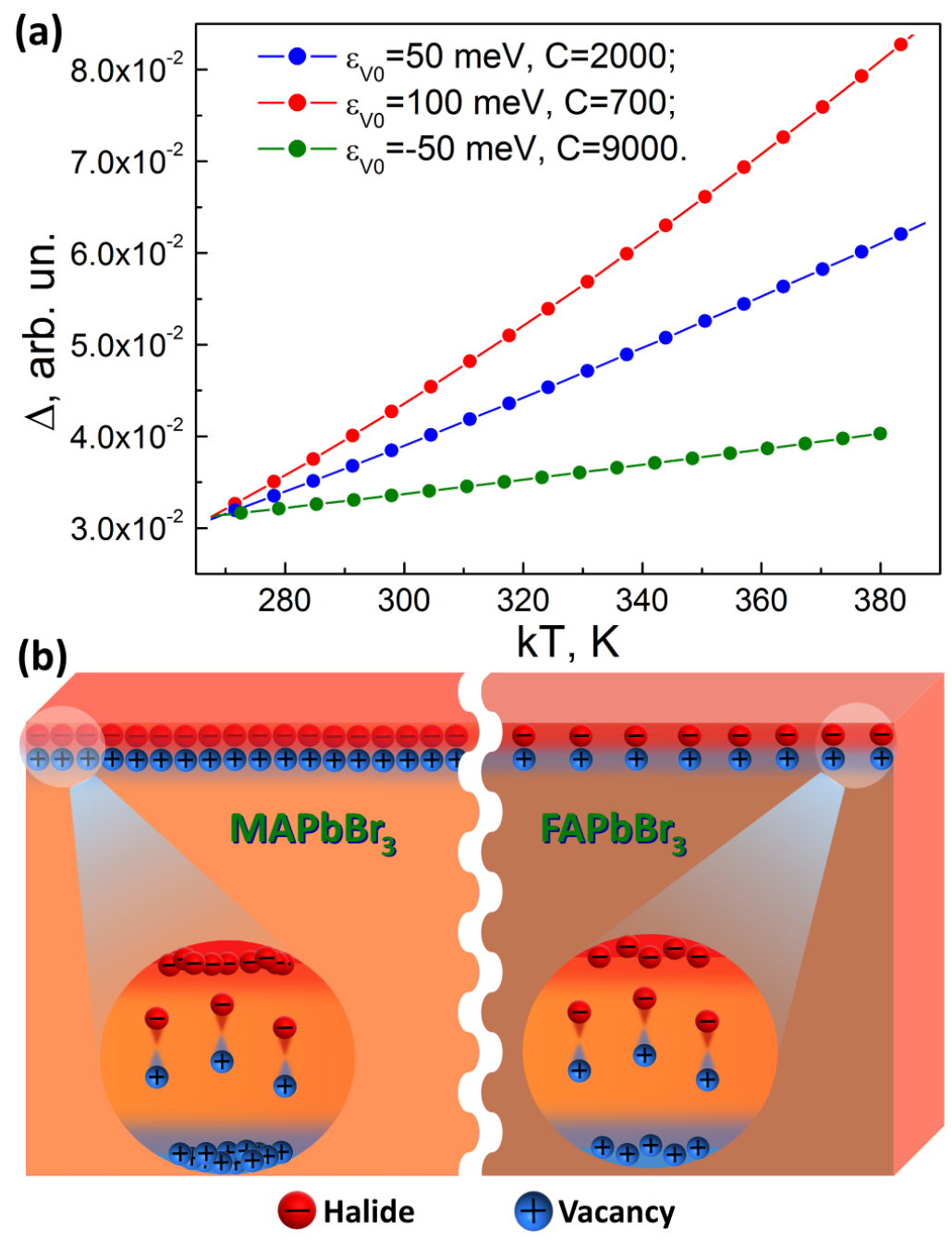

Figure 5. (a) The shift of the PL line obtained under $1 p$ excitation with respect to that obtained under $2 p$ excitation as a function of temperature calculated using Eq. (4) for three values of the vacancy formation energy $\varepsilon_{V_{0}}$; (b) Schematic diagram of electric field formation due to the spontaneous separation of negatively charged ions and positively charged vacancies in the perovskite single crystal.

where, $\varepsilon_{v 0}$ is the vacancy formation energy measured relative to the internal energy of halide ions on the surface, ${ }^{N_{v 0}}$ and $N_{s 0}$ are the concentrations of available sites for the halide vacancies in the bulk and halide ions on the surface, respectively. Substituting Eq. (3) into Eq. (2) we obtain 


$$
\Delta \propto \frac{(k T)^{5 / 3} \cdot \exp \left[-\frac{2 \varepsilon_{V 0}}{3 k T}\right]}{\left(C \cdot(k T)^{1 / 3} \cdot \exp \left[-\frac{\varepsilon_{V 0}}{3 k T}\right]+2 k T\right)}
$$

where the temperature-independent factor $\mathrm{C}$ depends on material parameters including the light penetration depth $x_{0}$, as shown by Eq. (S11) in the Supporting Information. According to Eq. (6), the effect of temperature on $\Delta$ depends on the vacancy formation energy $\varepsilon_{v 0}$ and always results in an increase of $\Delta$, i.e. increases the blue shift of the surface layer PL with respect to the bulk PL, as illustrated in Fig. 5. This behavior is in qualitative agreement with the experimental observation illustrated in Fig. 4. In Fig.S7, we plot the PL shift as a function of temperature for $\mathrm{MAPbBr}_{3}$ modeled using the equation shown in the inset. The PL line observed under twophoton excitation should have significantly lower shift (as seen from Eq. (3)) due to much longer light penetration depth $x_{0}$ ). It is worth pointing out that in general, there can be no visible correlation between the value of the blue shift at room temperature and the strength of its temperature dependence. This can be due to different values of ${ }^{\varepsilon_{v 0}}$ for different materials. In case if the binding energy of halide ions is higher on the surface then it is in the bulk the energy $\varepsilon_{v 0}$ can even be slightly negative (see green curve on Fig. 5(a) favoring generation of ion-vacancy pairs with ions placed on the crystal surface.

In conclusion, our theoretical analysis shows that the experimentally observed difference in the positions of PL line for one-photon and two-photon excitations is caused by the electric field generated in the surface layer of the crystals. This electric field is due to the spontaneous separation of negatively charged ions and positively charged vacancies (See Fig. 5(b)). This field causes an increase in the bandgap in the surface layer of the materials thus shifting the PL line to a higher energy. Indeed, the shift of the PL line with temperature predicted by our theory is 
qualitatively consistent with the experimental observations. The thickness of the surface layer is determined by the vacancy screening and typically is below $1 \mu \mathrm{m}$ suggesting that the properties of widely studied thin films of the perovskites are controlled by halide vacancies. The obtained experimental results and their theoretical interpretation provide a framework for understanding and improving the properties of the lead halide perovskite single crystals. Given that the performance of perovskite devices is limited by the quality of the surface layers, our findings will focus effort on the development of field-neutralizing procedures or surface passivation during crystal fabrication in order to achieve high-performance perovskite crystal devices.

\section{ASSOCIATED CONTENT}

\section{Supporting Information}

"This material is available free of charge via the Internet at http://pubs.acs.org." Theoretical analysis, details of all experimental procedures, and supporting figures.

\section{AUTHOR INFORMATION}

S.P.S, V.M.B and E.Y. contributed equally to the work

\section{Corresponding Author}

*E-mail: omar.abdelsaboor@kaust.edu.sa

\section{Notes}

The authors declare no competing financial interests.

\section{ACKNOWLEDGMENT}


King Abdullah University of Science and Technology (KAUST) supported the work reported here. The authors gratefully acknowledge funding support from KACST, Technology Innovation Center for Solid-State Lighting at KAUST.

\section{REFERENCES}

1. Xing, G.; Mathews, N.; Sun, S.; Lim, S. S.; Lam, Y. M.; Grätzel, M.; Mhaisalkar, S.; Sum, T. C. Science 2013, 342, (6156), 344-347.

2. $\quad$ Srimath Kandada, A. R.; Petrozza, A. Acc. Chem. Res.2016, 49, (3), 536-544.

3. $\quad$ Manser, J. S.; Christians, J. A.; Kamat, P. V. Chem. Rev. 2016.

4. $\quad$ Frost, J. M.; Butler, K. T.; Brivio, F.; Hendon, C. H.; van Schilfgaarde, M.; Walsh, A. Nano Lett. 2014, 14, (5), 2584-2590.

5. Abrusci, A.; Stranks, S. D.; Docampo, P.; Yip, H.-L.; Jen, A. K. Y.; Snaith, H. J. Nano Lett. 2013, 13, (7), 3124-3128.

6. Pan, J.; Quan, L. N.; Zhao, Y.; Peng, W.; Murali, B.; Sarmah, S. P.; Yuan, M.; Sinatra, L.; Alyami, N. M.; Liu, J.; Yassitepe, E.; Yang, Z.; Voznyy, O.; Comin, R.; Hedhili, M. N.; Mohammed, O. F.; Lu, Z. H.; Kim, D. H.; Sargent, E. H.; Bakr, O. M. Adv. Mater. 2016, 28, (39), 8718-8725.

7. Kojima, A.; Teshima, K.; Shirai, Y.; Miyasaka, T. J. Am. Chem. Soc. 2009, 131, (17), 6050-6051.

8. $\quad$ Xu, W.; Liu, L.; Yang, L.; Shen, P.; Sun, B.; McLeod, J. A. Nano Lett. 2016, 16, (7), 4720-4725.

9. Shi, D.; Adinolfi, V.; Comin, R.; Yuan, M.; Alarousu, E.; Buin, A.; Chen, Y.; Hoogland, S.; Rothenberger, A.; Katsiev, K.; Losovyj, Y.; Zhang, X.; Dowben, P. A.; Mohammed, O. F.; Sargent, E. H.; Bakr, O. M. Science 2015, 347, (6221), 519-522.

10. Saidaminov, M. I.; Abdelhady, A. L.; Murali, B.; Alarousu, E.; Burlakov, V. M.; Peng, W.; Dursun, I.; Wang, L.; He, Y.; Maculan, G.; Goriely, A.; Wu, T.; Mohammed, O. F.; Bakr, O. M. Nat. Commun. 2015, 6, 7586.

11. Zhumekenov, A. A.; Saidaminov, M. I.; Haque, M. A.; Alarousu, E.; Sarmah, S. P.; Murali, B.; Dursun, I.; Miao, X.-H.; Abdelhady, A. L.; Wu, T.; Mohammed, O. F.; Bakr, O. M. ACS Energy Lett. 2016, 1, (1), 32-37.

12. Tian, W.; Zhao, C.; Leng, J.; Cui, R.; Jin, S. J. Am. Chem. Soc 2015, 137, (39), 1245812461.

13. Peng, W.; Wang, L.; Murali, B.; Ho, K.-T.; Bera, A.; Cho, N.; Kang, C.-F.; Burlakov, V. M.; Pan, J.; Sinatra, L.; Ma, C.; Xu, W.; Shi, D.; Alarousu, E.; Goriely, A.; He, J.-H.;

Mohammed, O. F.; Wu, T.; Bakr, O. M. Adv. Mater. 2016, 28, (17), 3383-3390.

14. Murali, B.; Saidaminov, M. I.; Abdelhady, A. L.; Peng, W.; Liu, J.; Pan, J.; Bakr, O. M.; Mohammed, O. F. J. Mater. Chem. C 2016, 4, (13), 2545-2552.

15. Yang, Y.; Yan, Y.; Yang, M.; Choi, S.; Zhu, K.; Luther, J. M.; Beard, M. C. Nat. Commun. 2015, 6, 7961.

16. Leguy, A. M. A.; Hu, Y.; Campoy-Quiles, M.; Alonso, M. I.; Weber, O. J.; Azarhoosh, P.; van Schilfgaarde, M.; Weller, M. T.; Bein, T.; Nelson, J.; Docampo, P.; Barnes, P. R. F. Chem. Mater. 2015, 27, (9), 3397-3407. 
17. Gangishetty, M. K.; Scott, R. W. J.; Kelly, T. L. Nanoscale 2016, 8, (12), 6300-6307.

18. Yang, J.; Siempelkamp, B. D.; Liu, D.; Kelly, T. L. ACS Nano 2015, 9, (2), 1955-1963.

19. Mosconi, E.; Meggiolaro, D.; Snaith, H. J.; Stranks, S. D.; De Angelis, F. Energy Environ. Sci. 2016.

20. Azpiroz, J. M.; Mosconi, E.; Bisquert, J.; De Angelis, F. Energy Environ. Sci. 2015, 8, (7), 2118-2127.

21. Chen, B.; Yang, M.; Zheng, X.; Wu, C.; Li, W.; Yan, Y.; Bisquert, J.; Garcia-Belmonte, G.; Zhu, K.; Priya, S. J. Phys. Chem. Lett. 2015, 6, (23), 4693-4700.

22. Eperon, G. E.; Habisreutinger, S. N.; Leijtens, T.; Bruijnaers, B. J.; van Franeker, J. J.; deQuilettes, D. W.; Pathak, S.; Sutton, R. J.; Grancini, G.; Ginger, D. S.; Janssen, R. A. J.; Petrozza, A.; Snaith, H. J. ACS Nano 2015, 9, (9), 9380-9393.

23. Abate, A.; Saliba, M.; Hollman, D. J.; Stranks, S. D.; Wojciechowski, K.; Avolio, R.; Grancini, G.; Petrozza, A.; Snaith, H. J. Nano Lett. 2014, 14, (6), 3247-3254.

24. Mosconi, E.; Meggiolaro, D.; Snaith, H. J.; Stranks, S. D.; De Angelis, F. Energy Environ. Sci. 2016, 9, (10), 3180-3187.

25. Yakunin, S.; Protesescu, L.; Krieg, F.; Bodnarchuk, M. I.; Nedelcu, G.; Humer, M.; De Luca, G.; Fiebig, M.; Heiss, W.; Kovalenko, M. V. Nat. Commun.2015, 6, 8056.

26. Walters, G.; Sutherland, B. R.; Hoogland, S.; Shi, D.; Comin, R.; Sellan, D. P.; Bakr, O. M.; Sargent, E. H. ACS Nano 2015, 9, (9), 9340-9346.

27. Yamada, Y.; Yamada, T.; Phuong, L. Q.; Maruyama, N.; Nishimura, H.; Wakamiya, A.; Murata, Y.; Kanemitsu, Y. J. Am. Chem. Soc 2015, 137, (33), 10456-10459.

28. Yang, Y.; Yang, M.; Li, Z.; Crisp, R.; Zhu, K.; Beard, M. C. J. Phys. Chem. Lett. 2015, 6, (23), 4688-4692.

29. Ghosh, B.; Nahas, S.; Bhowmick, S.; Agarwal, A. Phys. Rev. B 2015, 91, (11), 115433.

30. Mak, K. F.; Lui, C. H.; Shan, J.; Heinz, T. F. Phys. Rev. Lett. 2009, 102, (25), 256405.

31. Rodrigo, G. A.; Xiaoliang, Z.; Saikat, M.; Ravindra, P.; Alexandre, R. R.; Shashi, P. K. Phys.: Condens. Matter 2013, 25, (19), 195801.

32. Harbeke, G. J. Phys. Chem. Solids 1963, 24, (7), 957-963.

33. Dennler, G.; Lungenschmied, C.; Sariciftci, N. S.; Schwödiauer, R.; Bauer, S.; Reiss, H. Appl. Phys. Lett.2005, 87, (16), 163501.

34. Cancellieri, C.; Fontaine, D.; Gariglio, S.; Reyren, N.; Caviglia, A. D.; Fête, A.; Leake, S. J.; Pauli, S. A.; Willmott, P. R.; Stengel, M.; Ghosez, P.; Triscone, J. M. Phys. Rev. Lett. 2011, 107, (5), 056102.

35. Frenkel, J. Zeitschrift für Physik 1926, 35, (8), 652-669.

36. Yuan, Y.; Huang, J. Acc. Chem. Res. 2016, 49, (2), 286-293.

37. deQuilettes, D. W.; Zhang, W.; Burlakov, V. M.; Graham, D. J.; Leijtens, T.; Osherov, A.; Bulović, V.; Snaith, H. J.; Ginger, D. S.; Stranks, S. D. Nat. Commun. 2016, 7, 11683.

38. Yang, T.-Y.; Gregori, G.; Pellet, N.; Grätzel, M.; Maier, J. Angew. Chem. Int. Ed 2015, 54, (27), 7905-7910.

39. Eames, C.; Frost, J. M.; Barnes, P. R. F.; O’Regan, B. C.; Walsh, A.; Islam, M. S. Nat. Commun. 2015, 6, 7497.

40. Yang, D.; Ming, W.; Shi, H.; Zhang, L.; Du, M.-H. Chem. Mater. 2016, 28, (12), 43494357.

41. Haruyama, J.; Sodeyama, K.; Han, L.; Tateyama, Y. J. Am. Chem. Soc.2015, 137, (32), 10048-10051. 
42. Fang, H.-H.; Adjokatse, S.; Wei, H.; Yang, J.; Blake, G. R.; Huang, J.; Even, J.; Loi, M. A. Sci. Adv 2016, 2, (7).

43. Yun, J. S.; Seidel, J.; Kim, J.; Soufiani, A. M.; Huang, S.; Lau, J.; Jeon, N. J.; Seok, S. I.; Green, M. A.; Ho-Baillie, A. Adv. Energy Mater. 2016, 6, (13), 1600330-n/a.

44. Shao, Y.; Fang, Y.; Li, T.; Wang, Q.; Dong, Q.; Deng, Y.; Yuan, Y.; Wei, H.; Wang, M.; Gruverman, A.; Shield, J.; Huang, J. Energy Environ. Sci. 2016, 9, (5), 1752-1759.

45. Murali, B.; Dey, S.; Abdelhady, A. L.; Peng, W.; Alarousu, E.; Kirmani, A. R.; Cho, N.; Sarmah, S. P.; Parida, M. R.; Saidaminov, M. I.; Zhumekenov, A. A.; Sun, J.; Alias, M. S.;

Yengel, E.; Ooi, B. S.; Amassian, A.; Bakr, O. M.; Mohammed, O. F. ACS Energy Lett. 2016, 1, (6), 1119-1126.

46. Murali, B.; Yengel, E.; Peng, W.; Chen, Z.; Alias, M. S.; Alarousu, E.; Ooi, B. S.;

Burlakov, V.; Goriely, A.; Eddaoudi, M.; Bakr, O. M.; Mohammed, O. F. J. Phys. Chem. Lett. 2016, 137-143. 